\title{
Atenção farmacêutica no contexto da estratégia de saúde da família
}

\author{
Aline Aparecida Foppa', Gabriela Bevilacqua ${ }^{1}$, Luciano Henrique Pinto ${ }^{2}$, Carine Raquel Blatt ${ }^{1 *}$ \\ ${ }^{1}$ Curso de Farmácia, Departamento de Ciências Farmacêuticas, departamento de Saúde Pública, Universidade \\ Federal de Santa Catarina, 'Programa de Residência Integrada em Saúde da Família, Departamento de Saúde \\ Pública, Universidade Federal de Santa Catarina
}

*Correspondência:

C. R. Blatt

Universidade Federal de Santa

Catarina - Campus Trindade

Curso de Farmácia

Departamento de Ciências

Farmacêuticas

88040-370 - Florianópolis - SC, Brasil

E-mail: carine.blatt@gmail.com
A assistência farmacêutica, no âmbito do Sistema Único de Saúde (SUS), é muitas vezes realizada com o objetivo primordial de garantir o abastecimento dos medicamentos nas Unidades Locais de Saúde. Freqüentemente, os cuidados com os pacientes e a promoção do uso racional dos medicamentos são marginalizados. Contudo, durante o desenvolvimento do seguimento farmacoterapêutico, são identificados problemas relacionados aos medicamentos (PRM) e dificuldades na adesão ao tratamento farmacológico, os quais estão relacionados a questões familiares e sociais, e têm contribuído para o não-sucesso terapêutico, portanto pontos-chave a serem considerados durante a Atenção Farmacêutica. Foi realizado o seguimento farmacoterapêutico de quatro pacientes atendidos em uma unidade de saúde do município de Florianópolis. Para a resolução dos PRMs, adotou-se abordagem que inclui os contextos familiar e social e, que utiliza ferramentas como o genograma e o ecomapa, além do trabalho interdisciplinar. Observou-se a importância da família e dos grupos de apoio para a resolução dos PRMs e a necessidade do farmacêutico de aprimorar a prática de atenção farmacêutica mediante a utilização de tais ferramentas. Percebeu-se, ainda, a importância das visitas domiciliares para conhecer melhor o ambiente familiar e a necessidade de trabalho interdisciplinar para melhorar a qualidade do atendimento.

\section{INTRODUÇÃO}

A assistência farmacêutica é um conjunto de ações desenvolvidas pelo farmacêutico e por outros profissionais de saúde voltadas à promoção, à proteção e à recuperação da saúde, tanto no nível individual como no coletivo, tendo o medicamento como insumo essencial (Ivama et al., 2002). No âmbito do Sistema Único de Saúde (SUS), a
Unitermos

- Atenção Farmacêutica

- Medicamentos/uso racional

- Saúde da Família

- Genograma

- Ecomapa assistência farmacêutica tem como objetivo primordial garantir o abastecimento contínuo e o uso racional de medicamentos nas Unidades Básicas de Saúde (UBS), mediante processos de seleção, programação, aquisição, armazenamento, prescrição e dispensação (Marin et al., 2003). Nesses processos, verifica-se com freqüência a concentração das ações no medicamento, marginalizando o cuidado com o paciente. 
A Atenção Farmacêutica, uma das atividades da Assistência Farmacêutica, engloba ações específicas do profissional farmacêutico no contexto da assistência ao paciente, que visam à promoção do uso racional de medicamentos (Ivama et al., 2002). É no momento em que o farmacêutico assume responsabilidades no cuidado com o paciente, por meio da prática da Atenção Farmacêutica, que são identificados inúmeros problemas relacionados aos medicamentos (PRM) e dificuldades na adesão ao tratamento farmacológico.

O seguimento farmacoterapêutico é a prática profissional em que o farmacêutico se responsabiliza pelas necessidades do paciente relacionadas com o medicamento, o qual se realiza mediante a detecção de PRMs para a prevenção e resolução de resultados negativos associados aos medicamentos (RNMs) (Comitê de Consenso, 2007).

O Terceiro Consenso de Granada (Comitê de Consenso, 2007) define PRM como situações em que o uso do medicamento, provoca um resultado negativo associado ao seu uso. Esse consenso propõe a classificação dos PRM em função dos requisitos que todo medicamento deve ter para ser utilizado: ser necessário, efetivo e seguro.

Para Cipolle, Strand e Morley (2000), os PRMs podem ser resolvidos ou prevenidos somente quando se conhecem claramente as causas do problema. Dessa forma, é fundamental identificar e classificar o problema e sua causa.

De modo geral, os artigos que discutem esse tema somente identificam os PRMs com causas relacionadas ao medicamento, ao paciente, ao dispensador e ao sistema sanitário. Entretanto, durante o seguimento farmacoterapêutico de pacientes que foram atendidos em "UBS" do município de Florianópolis, observou-se que algumas intervenções farmacêuticas para a resolução de PRMs, que seguiam a conduta até então proposta eram ineficazes. Na maioria dos casos, o paciente sozinho era incapaz de compreender ou de executar as intervenções, e por diversas vezes as resoluções desses problemas estavam relacionadas ao contexto familiar e/ou social.

Abandono, ausência de cuidador para ajudar no manejo dos medicamentos, cuidadores sem preparo adequado para lidar com determinada situação, necessidade de uma rede de apoio e falta de habilidade com conflitos familiares são exemplos de PRM que podem ser resolvidos com a intervenção junto à família. No entanto, como apontado anteriormente, tais fatores são geralmente excluídos no momento da determinação das causas de PRM e na formulação de intervenções.

Considerando o papel da família e da rede de apoio no processo de cuidado dos pacientes que apresentam dificuldades no uso dos medicamentos, o objetivo deste estudo é apresentar quatro casos de pacientes portadores de patologias crônicas em seguimento farmacoterapêutico, em que o trabalho em equipe interdisciplinar e a intervenção no contexto familiar e social, contribuíram para a resolução de PRMs e para o sucesso da terapia.

\section{METODOLOGIA}

Este trabalho foi realizado no decorrer das atividades de estágio obrigatório do Curso de Farmácia da Universidade Federal de Santa Catarina, na Unidade Local de Saúde (ULS) da Agronômica, no município de Florianópolis, Santa Catarina. Essa unidade de saúde, mediante convênio entre a Prefeitura Municipal de Florianópolis (PMF) e a Universidade Federal de Santa Catarina (UFSC), possui o Programa de Residência em Saúde da Família, com uma equipe de saúde formada por médico, enfermeiro, odontólogo, farmacêutico, psicólogo, nutricionista, assistente social, técnico de enfermagem e agente comunitário de saúde.

O método de estudo escolhido foi o do tipo estudo de caso, em que foram selecionados quatro casos cujos pacientes eram portadores de patologias crônicas: paciente $\mathrm{A}$ (hipertensão arterial, insuficiência cardíaca congestiva, hiperlipidemia e diabetes mellitus tipo II); paciente B (doença de Alzheimer); paciente C (doença de Parkinson, arritmia e insuficiência cardíaca congestiva); e paciente D (artrite reumatóide, depressão, hipotireoidismo, gastrite).

A coleta dos dados foi realizada por meio dos prontuários, visitas domiciliares e consultas farmacêuticas, realizadas entre os meses de setembro e dezembro de 2006. O seguimento farmacoterapêutico foi realizado pelo Método Dáder (Machuca, Fernández-Llimós, Faus, 2003) e pelo Segundo Consenso de Granada (Consensus Commitee, 2002).

O Método Dáder baseia-se na obtenção da história farmacoterapêutica do paciente, isto é, nos problemas de saúde que ele apresenta e nos medicamentos que utiliza, e na avaliação de seu estado de situação em uma data determinada, a fim de identificar e resolver os possíveis PRMs apresentados pelo paciente. Após essa identificação são realizadas as intervenções farmacêuticas necessárias para resolver os PRMs e, posteriormente, a avaliação dos resultados obtidos (Machuca, Fernández-Llimós, Faus, 2003).

Para documentar o contexto familiar e social de cada participante, foi elaborado o genograma, com base nos símbolos preconizados por Arruga (2001), e o ecomapa, com base no modelo preconizado por Sluzki (2003) e Cecagno, Souza e Jardim (2004).

O genograma é a representação gráfica da história de vida da família e da inter-relação biológica e afetiva entre seus membros. Conforme pode ser observado na Figura 1, consiste em um diagrama que detalha a estrutura e o histó- 


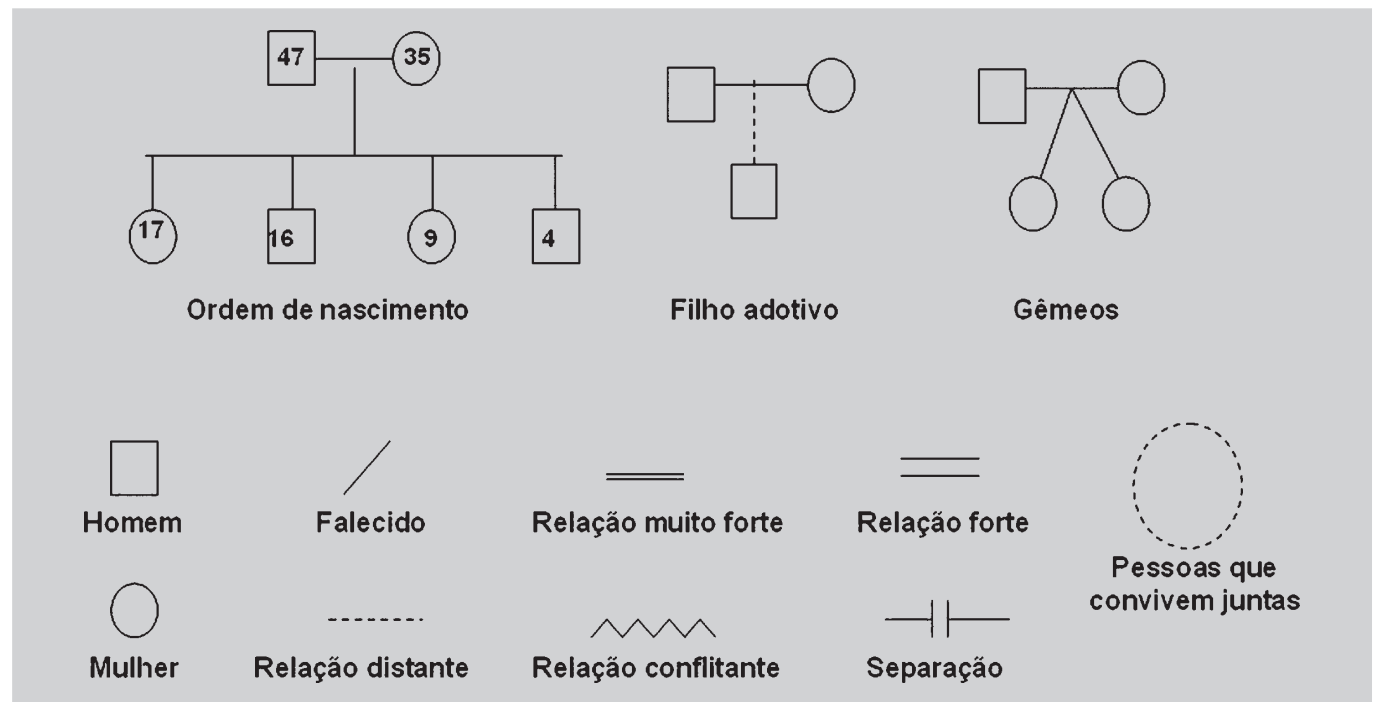

FIGURA 1 - Símbolos utilizados na construção do genograma (adaptado de Arruga, 2001).

rico familiar, e fornece informações sobre os vários papéis de seus membros e das diferentes gerações que compõem a família (Athayde, Gil, 2005).

Já o ecomapa reflete os relacionamentos dos membros da família com sistemas mais amplos, como amigos, trabalho, instituições e comunidade (More, 2005), os quais constituem a rede social pessoal (Sluzki, 2003, Wessels, 1991). A rede social pessoal pode ser registrada em forma de mapa mínimo, que inclui todos os indivíduos com quem interage determinada pessoa. Conforme pode ser observado na Figura 2, o mapa pode ser sistematizado em quatro quadrantes: família; amizades; relações de trabalho ou escolares; e relações comunitárias, de serviço ou de credo. Sobre esses quadrantes inscrevem-se três áreas: um círculo interno de relações íntimas, tais como familiares diretos, com contato cotidiano e amigos próximos; um círculo intermediário de relações pessoais com menor grau de compromisso, tais como relações sociais ou profissionais com contato pessoal mas sem intimidade; e um círculo externo de conhecidos e relações ocasionais (Wessels, 1991).

Após o levantamento das informações, todos os casos foram discutidos com a equipe de saúde, e as intervenções foram propostas durante a realização das visitas domiciliares e das consultas farmacêuticas.

O trabalho foi aprovado pelo Comitê de Ética em Pesquisas com Seres Humanos da Universidade Federal de Santa Catarina, de acordo com o protocolo n 302 de 2006.

\section{RESULTADOS E DISCUSSÃO}

Um breve resumo de cada caso é apresentado identificando-se a patologia do paciente e aspectos do contexto familiar e social, os quais também podem ser visualizados

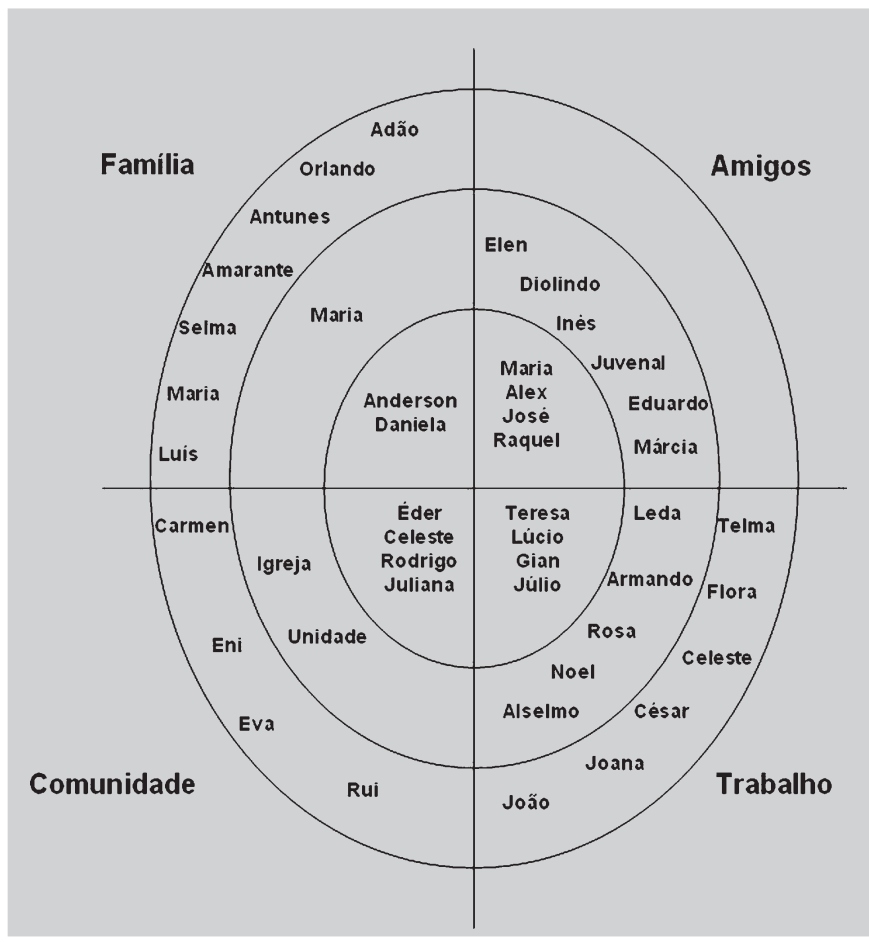

FIGURA 2 - Representação de um ecomapa (adaptado de Cecagno, Souza, Jardim, 2004).

a partir do genograma e do ecomapa. Em seguida, é realizada a descrição dos PRMs detectados, seguida das propostas de intervenções, da inserção delas no contexto familiar e da resolução dos casos.

Paciente A: do sexo feminino, viúva, 60 anos, reside sozinha e teve 8 gestações. A paciente mora ao lado da casa de dois de seus filhos (Filho 1 e Filho 3 ) e apresenta forte relação com a filha (Filho 2), porém esta passa pouco tem- 
po com a mãe devido ao trabalho. Os outros filhos não moram na mesma cidade. A paciente passa grande parte do seu tempo na casa dos Filhos 1 e 3, onde trabalha, realiza as refeições e ajuda a cuidar dos netos. A paciente freqüenta a igreja da comunidade, que é, segundo ela, um apoio muito importante em sua vida. Os problemas de saúde tratados pela paciente são hipertensão arterial, insuficiência cardíaca congestiva (ICC), hiperlipidemia e diabetes mellitus tipo II. Outros problemas identificados foram obesidade, sedentarismo, alimentação inadequada e cãibras.

A escolha da Paciente A se deu em decorrência de inúmeros fatores, sendo o principal a polimedicação. Além deste, há o início da insulinoterapia, a dificuldade no controle do peso e a possibilidade da inclusão da família na participação da terapia da paciente.

Relacionando as queixas apresentadas pela Paciente A e o tratamento medicamentoso, foram propostas intervenções que incluíam os membros da família para auxiliar na resolução dos possíveis PRMs, visto que o genograma e o ecomapa dela revelam que seus familiares constituem os principais membros da sua rede social significativa.

Conforme aponta Elsen (2004), a família é geralmente a primeira e a mais constante unidade de saúde para seus membros, e o cuidado prestado envolve ações de promoção de saúde, prevenção e tratamento de doenças, incluindo ações de reabilitação, que são baseadas na cultura e na interação com profissionais de saúde. Além disso, a participação da família na resolução dos problemas de saúde pode acelerar o tratamento e torná-lo menos dispendioso (Falceto, Fernandes, Wartchow, 2004).

A queixa mais antiga, que aparecia com relativa freqüência, eram as cãibras. Tal sintoma foi atribuído à administração de grande quantidade de diuréticos depletores de potássio, necessários para o tratamento da insuficiência cardíaca congestiva e da hipertensão. Caracterizou-se, então, a possibilidade da existência de um PRM de segurança.

Na tentativa de solucionar os quadros de cãibra apresentados pela paciente, foram diminuídas as doses dos diuréticos gastadores de potássio, porém a manutenção da pressão arterial foi comprometida e as doses foram reajustadas. Outra intervenção farmacoterapêutica poderia ser a reposição de potássio por meio de suplementos medicamentosos, porém, visto que a paciente fazia uso de inúmeros medicamentos, optou-se por realizar intervenções nutricionais para o aumento na ingestão de alimentos ricos em potássio.

As orientações alimentares foram fornecidas à paciente pelas equipes de nutrição e farmácia da unidade de saúde, porém não foram acatadas, uma vez que a paciente não realizava a compra e o preparo de suas refeições. Desse modo, verificou-se a necessidade de intervenção junto à família da paciente, a qual se mostrou mais eficaz, considerando a diminuição das cãibras e a perda de peso verificadas durante o acompanhamento.

O início da insulinoterapia foi responsável pelo aparecimento de PRMs de efetividade e de segurança (hiperglicemia e episódios de hipoglicemia, respectivamente). A primeira intervenção relacionada com a administração da insulina foi a orientação de como aplicá-la corretamente. Paralelamente, foi ressaltada à paciente a necessidade do cumprimento de todas as refeições diárias, a importância de seguir rigorosamente a dieta alimentar.

Seguidas as orientações, observou-se relativa melhora no quadro de hiperglicemia da paciente, porém persistiam alguns episódios esporádicos de hipoglicemia. Em um de seus

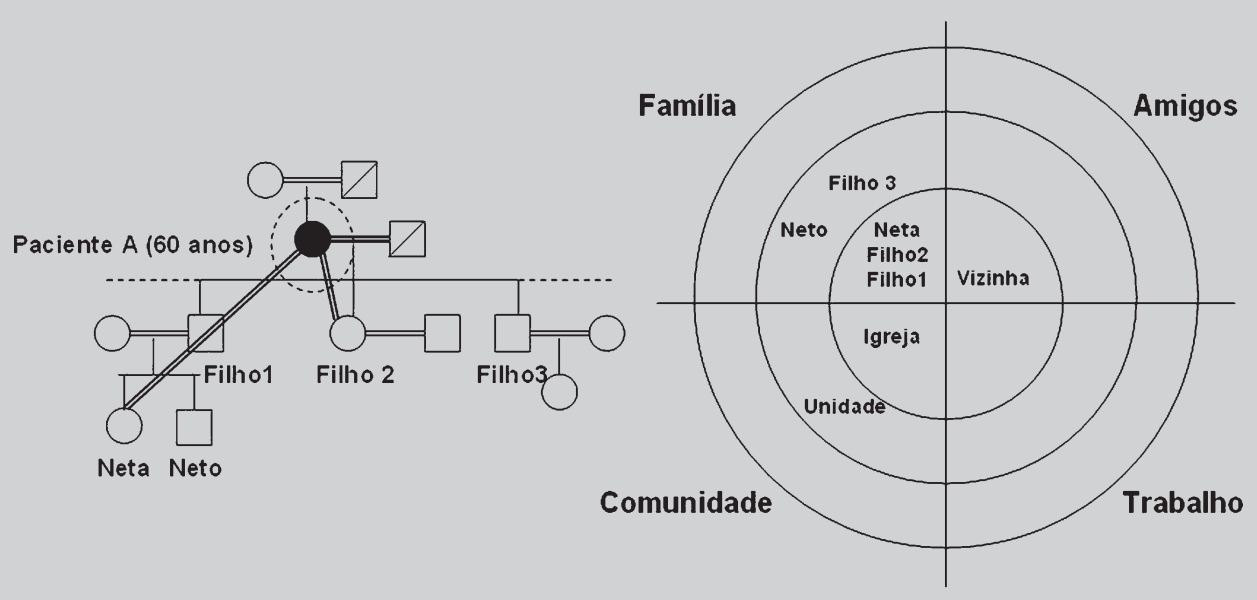

(1)

(2)

FIGURA 3 - Representação do genograma (1) e do ecomapa (2) da Paciente A. 
retornos à ULS, a paciente queixou-se de que não conseguia enxergar direito. Verificou-se que já havia sido indicado um oftalmologista para ela e que estava aguardando a consulta.

Com base nisso, percebeu-se, mais uma vez, que prestar orientações exclusivamente à paciente poderia não surtir o efeito desejado. Então, novamente, a intervenção realizada foi a orientação aos familiares quanto à detecção e ao controle dos sintomas das crises de hipoglicemia apresentados pela paciente, bem como em relação à vigília contínua de sua alimentação e da administração da insulina.

É importante enfatizar que, conforme aponta Caldas (1995), não se deve esperar que os cuidados sejam entendidos e executados corretamente sem que os responsáveis pelo paciente sejam orientados. Além disso, é fundamental que os profissionais de saúde treinem o cuidador e supervisionem a execução das atividades assistenciais até que a família se sinta segura para assumi-las.

Paciente B: sexo masculino, viúvo, 82 anos, reside com uma das filhas, com o genro e quatro netos. Essa família é responsável por todos os seus cuidados, uma vez que os demais filhos não moram na mesma cidade, Florianópolis. A filha não trabalha e dedica-se exclusivamente aos cuidados do pai, dos filhos e da casa. O paciente é acometido pela doença de Alzheimer, a qual se encontra em grau avançado, prejudicando sua capacidade de comunicação, locomoção e alimentação, bem como de executar outras atividades. Outros problemas identificados incluem desnutrição, edema em membros e perturbações do sono.

O paciente B já estava sendo acompanhado pela equipe de saúde da ULS, mediante visitas domiciliares, por apresentar dificuldades no deslocamento até a unidade em decorrência da doença. Durante as visitas domiciliares foram identificados PRMs e sobrecarga do cuidador, o que provocou sua inclusão no estudo, a fim de analisar a repercussão de tal demência no contexto familiar.

No momento do estudo, o problema de saúde em tratamento do paciente B era distúrbio do sono, sendo o ajuste do medicamento e da dose, o grande desafio para o sucesso dessa terapia, em virtude da idade avançada e do baixo peso do paciente. Tais fatores influenciam o aparecimento de PRMs de efetividade e de segurança (Machuca, Fernández-Llimós, Faus, 2003).

A primeira opção de tratamento para o distúrbio do sono foi amitriptilina $25 \mathrm{mg}$ administrada uma vez por dia. Após o início do tratamento, a cuidadora observou que esse medicamento causava no paciente sedação excessiva durante o dia, apesar de ser administrado à noite, o que dificultava ainda mais a realização das atividades diárias, caracterizando-se um PRM de segurança. Em discussão com a equipe, optou-se pela redução da dose para $12,5 \mathrm{mg} / \mathrm{dia}$, porém tal intervenção não foi suficiente para resolver o PRM. Desse modo, foi necessária a substituição do medicamento por um sedativo menos potente, o passiflorine.

No entanto, o passiflorine, segundo a cuidadora, se mostrou ineficaz, uma vez que o paciente voltou a apresentar dificuldades em dormir: acordava diversas vezes durante a noite. Diante desse PRM de efetividade, e para facilitar o ajuste da dose, o farmacêutico sugeriu a utilização de um medicamento com apresentação em gotas. A terapia foi alterada para clonazepan gotas, com dose inicial de 5 gotas/dia, podendo aumentar até 10 gotas/dia, ou diminuir a dose, conforme a resposta do paciente.

Todas essas intervenções só foram possíveis, aceitas e aplicadas devido à presença da cuidadora, neste caso a filha, uma vez que a doença de Alzheimer impossibilita seus

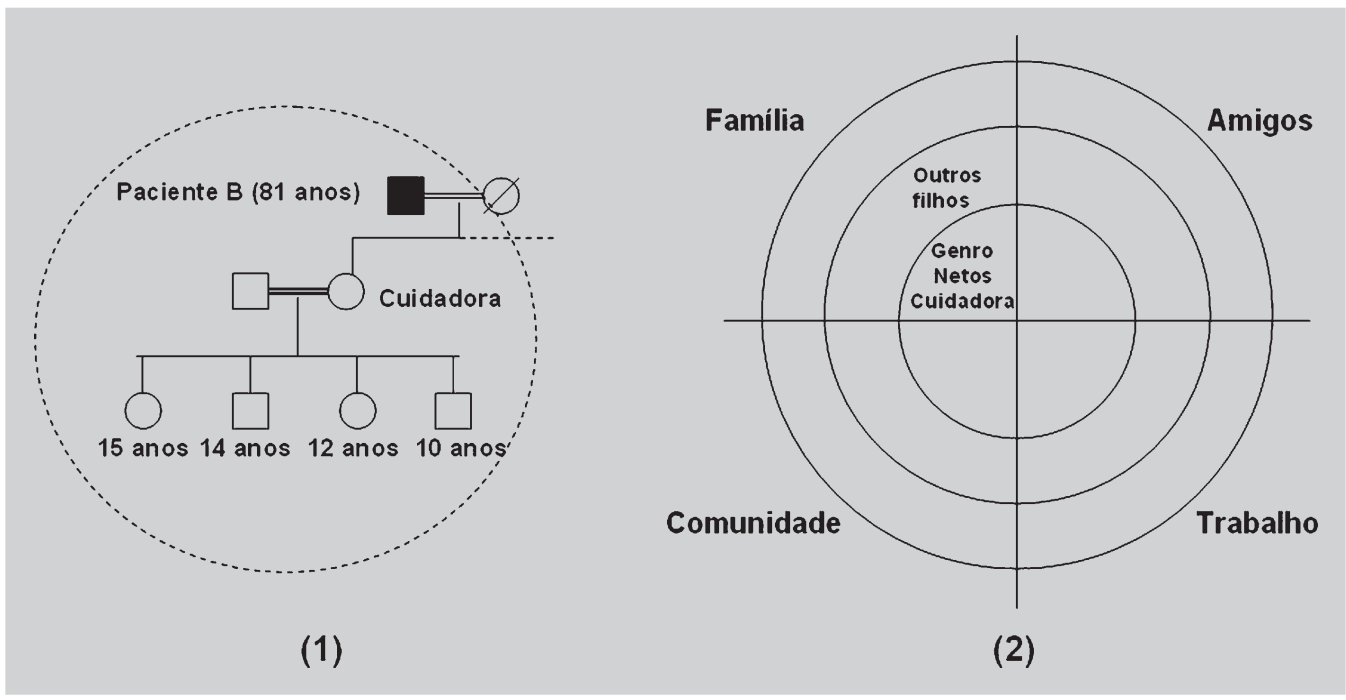

FIGURA 4 - Representação do genograma (1) e do ecomapa (2) do Paciente B. 
portadores de assumirem seus cuidados e de seguirem sozinhos a terapia proposta pela equipe de saúde.

Segundo Caldas (2003), quando um membro da família desencadeia um processo de dependência derivado de alguma doença, há uma alteração na dinâmica familiar. À medida que a pessoa vai desenvolvendo a doença, há uma mudança de papéis entre os membros da família, a fim de designar um deles para assumir a responsabilidade pelos cuidados do doente. Dessa maneira, o cuidador é a pessoa que chama a si a incumbência de realizar as tarefas que não são mais possíveis de ser executadas pelo doente (Karsch, 2003).

A doença de Alzheimer pode ser considerada uma doença familiar, por repercutir profundamente no cotidiano das famílias, especialmente para o familiar responsável em prover os cuidados no domicílio. Por isso, este deve receber atenção especial dos profissionais de saúde, tendo em vista que, na maioria das vezes, desconhece as condutas adequadas diante das manifestações das doenças e das exigências de cuidar do idoso fragilizado (Luzardo, Waldman, 2004).

No caso estudado, a cuidadora demonstrou, em diversos momentos, estar sobrecarregada e, em alguns momentos, relatou que a doença do pai influencia nas relações de sua família. Com o intuito de melhorar a qualidade de vida da cuidadora, e, conseqüentemente do próprio paciente, propôsse a inclusão dela no grupo de apoio da Associação Brasileira de Alzheimer, localizada no Hospital Universitário. Essa associação congrega familiares e profissionais que, através de suas vivências e conhecimentos, desenvolvem ações concretas de suporte emocional e a atualização a respeito de diagnóstico e do tratamento da doença.

Paciente C: sexo feminino, 86 anos, não é natural de
Florianópolis, porém reside nessa cidade há mais de 50 anos, período no qual passou a trabalhar e a morar na casa de uma família que atualmente se responsabiliza por seus cuidados. Durante esse tempo perdeu totalmente o contato com seus parentes. Na casa onde a paciente reside moram a cuidadora principal, que tem mais ou menos 50 anos de idade, o pai desta, que há poucos meses sofreu um derrame e também necessita de cuidados, e uma sobrinha da cuidadora, que ajuda em algumas tarefas da casa e nos cuidados com a paciente. Durante o acompanhamento verificou-se a sobrecarga da cuidadora. Os problemas de saúde apresentados pela paciente são: doença de Parkinson, arritmia e Insuficiência Cardíaca Congestiva. A paciente ainda se queixa de labirintite, dores nas pernas e insegurança em realizar as atividades diárias, necessitando de auxílio praticamente integral da cuidadora.

A escolha do caso se deu após uma visita domiciliar solicitada pela cuidadora, em decorrência da impossibilidade de se conseguir a medicação, pela não-padronização e pela dificuldade em deslocar a paciente até à ULS, em conseqüência de sua doença. Com a realização da visita visualizou-se o desconhecimento da doença e o isolamento social da paciente e de sua cuidadora, verificando-se a oportunidade da inclusão da paciente no presente estudo, para analisar a importância da rede de apoio no amparo a indivíduos parkinsonianos.

A paciente relatou episódios de tontura, os quais atrapalhavam as atividades diárias e aumentavam sua insegurança em realizá-las. Para minimizar tais sintomas, a cuidadora administrava por iniciativa própria o medicamento dimenidrato à paciente.

Como resultado da pesquisa realizada na literatura, verificou-se que tal sintoma poderia estar relacionado com:

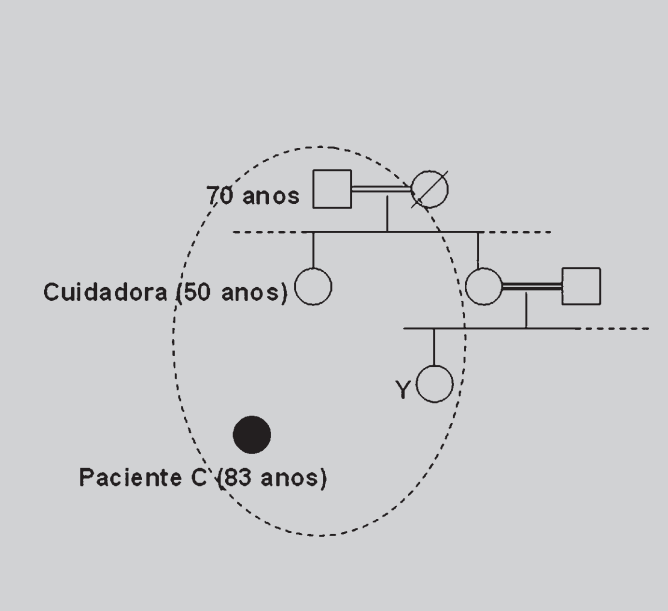

(1)

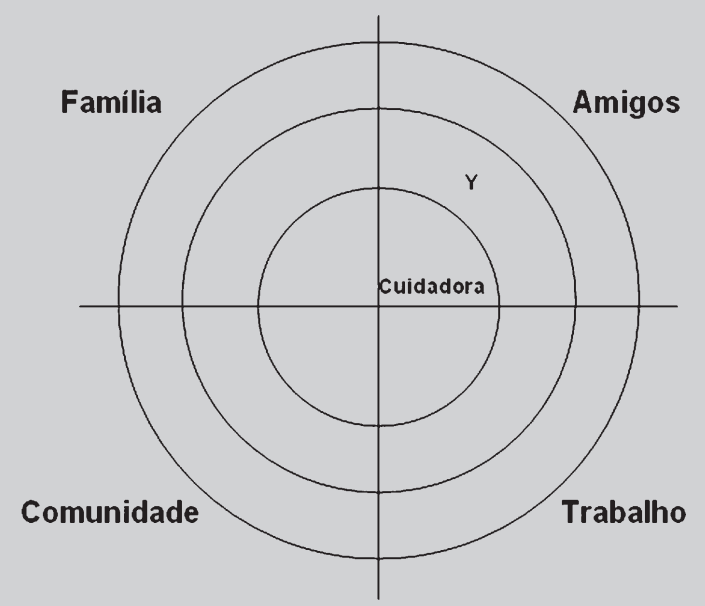

(2)

FIGURA 5 - Representação do genograma (1) e do ecomapa (2) da Paciente C. 
efeito de algum medicamento utilizado pela paciente como a levedopa/carbidopa e/ou enalapril; desidratação que ocorre principalmente em pacientes mais idosos e em estado de desnutrição doença cardíaca associada; e ação da própria doença, que pode causar sensações de cabeça vazia ou de tonturas vagas, geralmente associadas a escurecimento visual quando o paciente levanta-se.

Outra queixa que persistiu durante as visitas domiciliares foram as dores musculares, principalmente nos membros inferiores. Para amenizar tal sintoma, a cuidadora administrava à paciente o fitoterápico castanha da índia.

As principais manifestações clínicas da doença incluem tremores, rigidez muscular, hipocinesia e bradicinesia, depressão, distúrbios do sono, distúrbios cognitivos, da fala e respiratórios, dificuldades urinárias, tonturas e dores. Assim, além de medicamentosos, outros tratamentos complementares, como a fisioterapia, a terapia ocupacional e a fonoaudiologia, são considerados indispensáveis para minimizar essas manifestações e facilitar a realização de atividades diárias (Limongi, 2007).

Inicialmente, as causas propostas para ambas as queixas estavam relacionadas aos medicamentos, como reação adversa e inefetividade, porém se percebeu que o desconhecimento da doença e a falta de terapias complementares poderiam ser fatores significativos para a resolução dessas queixas. Assim, intervenções que possibilitassem à paciente conhecer melhor sua doença, além de tratamentos complementares e a troca de experiências com outros portadores, poderiam ser fundamentais na superação de dificuldades e insegurança decorrentes da doença. Tais intervenções poderiam ser conseguidas com a inserção da paciente no Grupo de Ajuda Mútua aos Portadores da Doença de Parkinson e seus Familiares, que se localiza no campus universitário e que conta com atividades físicas, apoio psicológico, fonoaudiologia e ajuda mútua.

Cuidar do idoso em casa é, com certeza, uma situação que deve ser preservada e estimulada, todavia, como aponta Karsch (2003), cuidar de um indivíduo idoso e incapacitado durante 24 horas sem pausa não é tarefa para uma mulher sozinha, com mais de 50 anos, sem apoios nem serviços que possam atender a suas necessidades, e sem uma política de proteção para o desempenho desse papel.

O cuidador familiar de idosos incapacitados precisa receber orientação de como proceder nas situações mais difíceis e, também, receber em casa visitas periódicas de profissionais (Karsch, 2003, Nakatani et al., 2003). Porém, isso requer que o profissional conheça a família, seus valores, crenças e visão de mundo que influenciam suas formas de cuidar. A partir daí poderá orientar e assistir o paciente e a família de uma maneira mais adequada e integrada à suas necessidades e a sua cultura (Resta, Budó,
2004). Somente mediante o acompanhamento e visitas domiciliares que esse conhecimento e essa aproximação tornam-se possíveis.

Além disso, em momentos de crise ou em situações de enfermidades, pode ser de extrema importância a mobilização de amigos, vizinhos e instituições, a fim de formar uma rede de apoio no amparo aos doentes e suas famílias (Falceto, Fernandes, Wartchow, 2004). Desse modo, o apoio social tem função mediadora, aumenta a vontade de viver e a auto-estima do paciente, o que contribui com o sucesso do tratamento (Minkler, 1985). Associações voluntárias e grupos de apoio também têm papel fundamental na tentativa de ativar e estreitar as redes sociais, evitando o isolamento desses indivíduos e, assim, melhorando as condições de saúde (Andrade, Vaitsman, 2002).

Paciente D: sexo feminino, 50 anos, reside em Florianópolis há cerca de 30 anos, teve 3 fillhos em 2 casamentos. Atualmente, a paciente mora com os dois filhos mais novos e com um companheiro, com quem convive há dois anos e com quem possui ótima relação. Seus problemas de saúde são: artrite reumatóide, depressão, hipotireoidismo e gastrite. As principais queixas da paciente, que fizeram com que ela procurasse os serviços da ULS, foram dificuldade de locomoção e dores contínuas.

Inicialmente, a paciente procurou o serviço de farmácia da ULS para sanar dúvidas a respeito de prescrições de diferentes médicos para tratar a mesma queixa. Ausência das posologias nas prescrições, prescrições mal compreendidas, desconhecimento da finalidade de alguns medicamentos e receio em relação à utilização de medicamentos controlados eram algumas das dúvidas da paciente. Tais dúvidas poderiam provocar a não-adesão ao tratamento e trazer como conseqüência o não-controle de seus problemas de saúde.

Após a análise do caso, pôde-se suspeitar, além do problema de adesão, a existência de PRM de necessidade, acarretando o não-controle da artrite reumatóide. A paciente fazia uso de antiinflamatórios não-esteroidais para o controle da artrite reumatóide e das fortes dores, porém estes não estavam sendo suficientes.

Diante das dúvidas e confusões geradas por prescrições médicas incompletas e duplicadas, e, também, do nãocontrole da doença, evidenciado pelas queixas da paciente, a primeira intervenção foi o encaminhamento ao médico da Unidade para avaliação do quadro geral da paciente. Um dos resultados dessa intervenção foi a prescrição de novo tratamento que objetivava amenizar as dores, além do encaminhamento ao reumatologista, para reavaliação do controle efetivo da doença. O especialista prescreveu metotrexato e, percebendo a relação próxima entre a paci- 


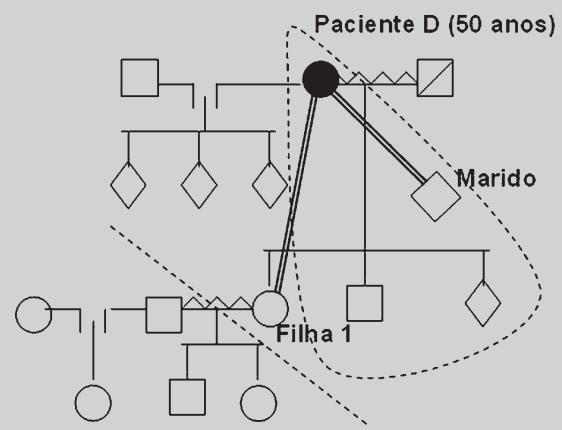

(1)

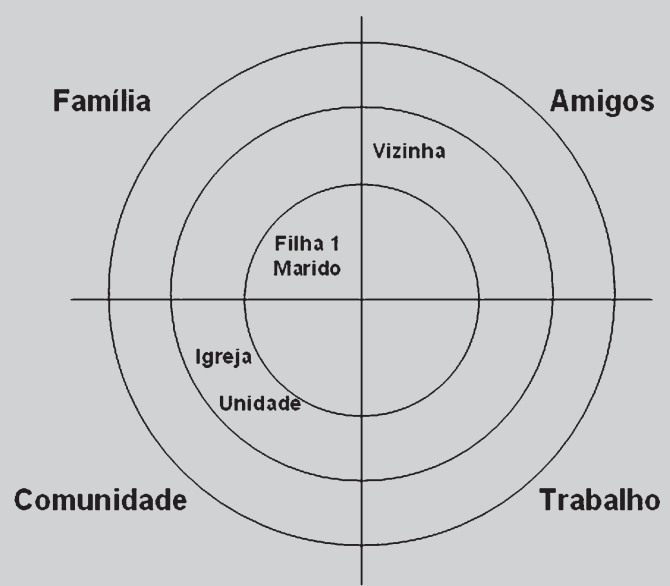

(2)

FIGURA 6 - Representação do genograma (1) e do ecomapa (2) da Paciente D.

ente e o farmacêutico, requereu a este o acompanhamento da nova terapia para verificar a eficácia e segurança, por meio do recordatório. Esse novo tratamento demonstrou melhora significativa nas dificuldades de locomoção e, quanto às dores apresentadas pela paciente, sem a ocorrência de efeitos adversos.

Durante a realização das consultas farmacêuticas descobriu-se a existência de problemas nas relações familiares. Devido a esse fato, a paciente foi incluída no estudo, a fim de avaliar se o contexto familiar também estaria influenciando na não-adesão da paciente ao tratamento.

A Paciente $\mathrm{D}$ teve algumas particularidades quanto à elaboração de seu genograma, pois uma abordagem inicial muito superficial das relações familiares foi insuficiente para expor problemas relevantes no acompanhamento do caso.

Inicialmente, observaram-se problemas no relacionamento com seus dois primeiros maridos, com relatos de agressão, de etilismo e de proibição da paciente de trabalhar. Verificou-se, também, a forte relação com uma de suas filhas, bem como o ótimo relacionamento com o atual companheiro, o qual sempre a acompanhava nas consultas.

Em segundo momento, investigaram-se mais profundamente as outras relações familiares, seguindo sugestão fornecida pela psicóloga da unidade. Percebeu-se, então, que a Filha 1 da paciente apresentava relacionamento bastante conturbado com seu marido, semelhante ao quadro vivido pela mãe anos antes, uma vez que o genro não permitia que a filha trabalhasse. Além disso, descobriu-se que a enteada de sua filha tinha envolvimento com drogas. Essa investigação mais minuciosa foi decisiva para determinar as intervenções farmacêuticas desse caso.

O quadro de depressão tratado pelo médico com amitriptilina, após a análise inicial e superficial das rela- ções familiares, foi atribuído aos problemas das relações conjugais do passado. Assim, por se tratar de trauma, nenhuma intervenção foi realizada inicialmente. Após investigação aprofundada das relações familiares e a percepção de que problemas atuais, envolvendo sua filha, afetavam emocionalmente a paciente, propôs-se a integração desta e da filha ao grupo de apoio familiar na própria ULS, para que o suporte oferecido pelo grupo ajudasse na reconfiguração das relações familiares e contribuísse para o sucesso da terapia medicamentosa da depressão.

A família deve ser preparada para lidar com os sentimentos de culpa, frustração, raiva, depressão e outros que acompanham essa responsabilidade, uma vez que o paciente sente-se incomodado por causar transtorno e problemas aos familiares. Ao mesmo tempo, a sobrecarga física, emocional e econômico-financeira do cuidador de um familiar é imensa. Nesses casos, a atuação em conjunto com psicólogos e assistentes sociais é importante, pois permite suporte mais adequado à família.

\section{CONSIDERAÇÕES FINAIS}

Até há pouco tempo, o foco da Assistência Farmacêutica era basicamente restrito ao medicamento. Atualmente, tem-se buscado conciliar as ações voltadas ao medicamento e ao usuário. O desenvolvimento da Atenção Farmacêutica, no Brasil, possibilitou novo olhar ao cuidado no exercício da profissão farmacêutica, voltando a atenção ao usuário e à identificação, prevenção e resolução de problemas relacionados aos medicamentos. Com o presente trabalho verificou-se ser necessária a ampliação desse foco para abordagem mais ampla, que envolva os contextos familiar e social. 
Na prática do atendimento farmacêutico na atenção primária, verificou-se que a família pode ser um campo de atuação do farmacêutico para resolver de forma mais eficaz os problemas com a medicação de determinados pacientes, principalmente idosos, os quais, muitas vezes, apresentam doenças crônicas e/ou degenerativas e fazem uso de polimedicação.

Um estudo conduzido por Karsch (2003) demonstrou que cerca de $40 \%$ dos indivíduos com 65 anos ou mais de idade, precisam de algum tipo de ajuda para realizar pelo menos uma tarefa, como fazer compras, cuidar das finanças, preparar as refeições e limpar a casa. Uma parcela menor, $10 \%$, requer auxílio para realizar tarefas básicas, como tomar banho, vestir-se, ir ao banheiro, alimentar-se, sentar e levantar de cadeiras e camas. Assim, nesse processo de cuidado/cura, o cuidador desenvolve um papel muito significativo, constituindo muitas vezes a mais importante fonte de suporte aos idosos. Cerca de $80 \%$ a $90 \%$ dos serviços e cuidados recebidos por esses são dispensados pelos familiares (Silva, Neri, 2003). Dessa forma, verifica-se que a família tem papel determinante no processo de cuidar/curar, não podendo ser excluída no momento da determinação das causas e da realização das intervenções para a resolução de PRMs.

O profissional farmacêutico não faz parte da equipe mínima da ESF, contudo, conforme aponta Veber (2005), existem pelo menos três possibilidades de atuação desse profissional: no âmbito gerencial/administrativo; nas ações voltadas diretamente ao usuário do sistema de saúde que englobam a atividade de dispensação orientada de medicamentos, passando por atividades educativas de farmacovigilância e de Atenção Farmacêutica e nas atividades direcionadas aos outros profissionais da equipe de trabalho, destacando o suporte informativo em relação aos medicamentos, o auxílio na terapêutica e a contribuição para um tratamento medicamentoso seguro e eficaz.

Evidentemente que, para cada uma dessas áreas de atuação, o farmacêutico deve aprimorar seus conhecimentos. Nas ações relacionadas ao cuidado do paciente, além da prática de Atenção Farmacêutica, faz-se necessário o desenvolvimento de habilidades que envolvam o trabalho em equipe e a utilização de ferramentas que facilitem a abordagem familiar, a exemplo do genograma e do ecomapa, ambos utilizados neste trabalho para a resolução dos PRMs.

Conforme apontado por More (2005) e por Nakatani et al. (2003), o genograma tem função valiosa na identificação de recursos familiares que possam apoiar as intervenções sobre os problemas do paciente, sendo instrumento imprescindível para o estudo de famílias que possuem membros imobilizados, padecem de doenças crônicas ou encontram-se em estado terminal, uma vez que, junto com os dados estruturais, são abordados também dados mais específicos, como a situação do cuidador principal e dos cuidadores secundários. Dessa maneira, o genograma configura-se, provavelmente, como o instrumento-chave para se iniciar a orientação dos pacientes no modelo proposto atualmente para a atenção primária no Brasil. Além disso, todos os profissionais (médicos, enfermeiros, farmacêuticos e assistentes sociais) podem construir o genograma, dando maior ênfase em determinados aspectos do diagrama, conforme os objetivos que se deseja alcançar e as peculiaridades de suas atividades.

O ecomapa pode ajudar a avaliar os apoios e suportes disponíveis e sua utilização pela família. A colaboração de grupos de apoio e/ou associações de caráter voluntário pode ser valiosa para a promoção ou recuperação da saúde, mediante o fortalecimento das relações sociais do indivíduo e/ou de sua família. Essas instituições promovem uma oportunidade para a troca de conhecimento e cooperação entre seus participantes (Minkler, 2002). Dessa maneira, o levantamento de grupos de apoio disponíveis e/ou próximos à comunidade pela equipe de saúde torna-se muito importante, porque eles fornecem apoio tanto aos pacientes como, e, principalmente, aos cuidadores e familiares, contribuindo de forma significativa para a melhora do estado de saúde do paciente.

Utilizando ferramentas como o genograma e o ecomapa, integrando a resolução de PRMs com os contextos familiar e social, o farmacêutico atuará de forma mais coerente com a proposta atual da atenção primária do Sistema Único de Saúde, que visa à integralidade da assistência por meio do desenvolvimento de um trabalho com a família, à co-responsabilidade no cuidado, à formação de vínculo com o paciente e à troca de informações e experiências com os demais profissionais, de forma interdisciplinar.

O trabalho interdisciplinar é, atualmente, uma das bases para a mudança do modelo de assistência à saúde. No decorrer deste estudo, percebeu-se, principalmente durante os estudos de caso dos Pacientes A e B, a importância da contribuição de outros profissionais da equipe de saúde para a resolução de PRMs e para a melhora da qualidade de vida dos pacientes. Além disso, a realização de visitas domiciliares mostrou-se fundamental para conhecer melhor o ambiente familiar e para facilitar a identificação de problemas que possam interferir no sucesso do plano terapêutico.

Entretanto, é preciso destacar que, embora o cuidado familiar seja um aspecto importante, ele não se aplica a todos os idosos. Existem idosos que não têm família. Há outros cujas famílias são muito pobres ou cujos familiares 
precisam trabalhar e não podem deixar o mercado de trabalho para cuidar deles.

Além disso, essa abordagem familiar somente é possível em virtude da organização da atenção primária adotada atualmente no Brasil, denominada de Estratégia de Saúde da Família, a qual, conforme apontam Falceto, Fernandes e Wartchow (2004), está estruturada na forma de equipes multidisciplinares, que têm como foco a família, compreendida a partir do ambiente em que vive e de sua realidade, o que permite um entendimento ampliado do processo saúde/doença, bem como intervenções com maior significado social.

\section{ABSTRACT \\ Pharmaceutical care in the context of the family health strategy}

The main objective of pharmaceutical policy management within the scope of SUS (Brazilian National Health System) is to guarantee the supply of drugs to local health centers, therefore displacing patient care When drugrelated problems (DRP) and difficulties in adhering to the prescribed treatment are identified during the development of pharmaceutical follow-up, family and social issues have been shown to be reasons for unsuccessful treatment. They are key points to be considered during Pharmaceutical Care. The methodology chosen for this study was the pharmaceutical care of four patients monitored in a Health Unit in the city of Florianópolis. To solve drug related problems an approach was adopted that included family and social contexts using tools such as genogramming, ecomapping and interdisciplinary work. Family and support groups were shown to be important in addressing DRPs and there appears to be a need for the pharmacist to improve Pharmaceutical Care Practice by using these tools. The importance of home visits to gain a better understanding of the family environment was also observed, as well as the necessity of interdisciplinary work to improve the quality of care.

UNITERMS: Pharmaceutical Care. Drugs/rational use. Family Health Care. Genogram. Ecomap.

\section{REFÊRENCIAS BIBLIOGRÁFICAS}

ANDRADE, G. R. B.; VAITSMAN, J. Apoio social e redes: conectando solidariedade e saúde. Ciênc. Saúde Coletiva, Rio de Janeiro, v.7, n.4, p.925-934, 2002.

ARRUGA, I. Y. Atención a la familia: otra forma de enfocar los problemas de salud en atención primaria. Instrumentos de abordaje familiar. An.Sist. Sanit. Navar., Navarra, v.24, supl. 2, p.73-82, 2001.

ATHAYDE, E. S.; GIL, C. R. R. Possibilidades do uso do genograma no trabalho cotidiano dos médicos das equipes de saúde da família de Londrina. Revista Espaço para a Saúde, Londrina, v.6, n.2, p.13-22, 2005.

CALDAS, C. P. A abordagem do enfermeiro na assistência ao cliente portador de demência. Rev.Enferm. UERJ, Rio de Janeiro, v.3, n.2, p.209-216, 1995.

CALDAS, C. P. Envelhecimento com dependência: responsabilidades e demandas da família. Cad. Saúde pública, Rio de Janeiro, v.19, n.3, p.773-781, 2003.

CECAGNO, S.; SOUZA, M. D.; JARDIM, V. M. R. Compreendendo o contexto familiar no processo saúdedoença. Acta Sci., Health Sci., Maringá, v.26, n.1, p.107$112,2004$.

CIPOLLE, R. J.; STRAND, L. M.; MORLEY, P. C. $E l$ ejercicio de la atención farmacêutica. Madrid: McgrawRio, 2000. 369 p.

COMITÊ DE CONSENSO. Tercer Consenso de Granada sobre Problemas Relacionados con Medicamentos (PRM) y Resultados Negativos Asociados a la Medicación (RNM). Ars Pharm., Granada, v.48, n.1, p.5-17, 2007.

CONSENSUS COMMITEE. Segundo Consenso de Granada sobre Problemas Relacionados com Medicamentos. Ars Pharm., Granada, v.43, n.3-4, p.175-184, 2002.

FALCETO, O. G.; FERNANDES, C. L. C.; WARTCHOW, E. S. O médico, o paciente e sua família In: DUNCAN, B. B.; SCHIMIDT, M. I.; GILGLIANI, E. R. J. Medicina ambulatorial: condutas em Atenção Primária baseada em evidências. 3. ed. Porto Alegre: Artmed, 2004. p.115-124. 
IVAMA, A. M.; NOBLAT, L.; CASTRO, M. S.; OLIVEIRA, N. V. B. V.; JARAMILLO, N. M.; RECH, N.Consenso Brasileiro de Atenção Farmacêutica. Brasília: Organização Pan-Americana da Saúde, 2002. 24 p.

KARSCH, U. M. Idosos dependentes: famílias e cuidadores. Cad. saúde pública, Rio de Janeiro, v.19, n.3, p.861-866, 2003.

LIMONGI, J. C. P. Parkinson On Line. Disponível em: <http:/ /www.parkinson.med.br/>. Acesso em: 15 jan.2007.

LUZARDO, A. R.; WALDMAN, B. F. Atenção ao familiar cuidador do idoso com doença de Alzheimer. Acta sci., Health sci. Maringá, v.26, n.1, p.135-145, 2004.

MACHUCA, M.; FERNÁNDEZ-LLIMÓS, F.; FAUS, M. J. Método Dáder: manual de acompanhamento farmacoterapêutico. Granada: GIAF-UGR, 2003. 45 p.

MARIN, N.; LUIZA, V. L.; CASTRO, C. G. S. O.; SANTOS, S. M. Assistência Farmacêutica para gerentes municipais. Brasília: Organização Pan-Americana da Saúde/Organização Mundial da Saúde, 2003. 373 p.

MORE, C. L. O. O. As redes pessoais significativas como instrumento de intervenção psicológica no contexto comunitário. Paidéia, Ribeirão Preto, v.15, n.31, p.287297, 2005.
NAKATANI, A. Y. K.; SOUTO, C. C. S.; PAULETTE, L. M.; MELO, T. S.; SOUZA, M. M. Perfil dos cuidadores informais de idosos com déficit de autocuidado atendidos pelo Programa de Saúde da Família. Rev.Eletrôn.Enferm., Goiânia, v.5 n.1, 2003.

RESTA, D. G.; BUDÓ, M. L. D. A cultura e as formas de cuidar em família na visão de pacientes e cuidadores domiciliares. Acta sci., Health sci., Maringá, v.26, n.1, p.53-60, 2004.

SILVA, E. P. N.; NERI, A. L. Questões geradas pela convivência com idosos: indicações para programas de suporte familiar. In: NERI, A. L. (Org.). Qualidade de vida e idade madura. Campinas: Papirus, 1993. p.213236.

SLUZKI, C. E. A rede social na pratica sistêmica: alternativas terapêuticas. 2. ed. São Paulo: Casa do Psicólogo, 2003. p.37-85.

VEBER,A. P. A atuação do farmacêutico na saúde da família. In: CORDEIRO, B. C.; LEITE, S. N.(Org.). $O$ farmacêutico na Atenção à Saúde. Itajaí: Ed. da Univali, 2005. p.53-70.

WESSELS, B. Organizing capacity of societies and modernity. In: DETH, J. V.(Org.). Private groups and public life: social participation, voluntary association and political involvement in representative democracies. Londres: Jan van Deth, 1991. p.198-219.

Recebido para publicação em 28 de janeiro de 2008 Aceito para publicação em 14 de março de 2008 